Chirurg 2018 $89: 735$

https://doi.org/10.1007/s00104-018-0701-7

Online publiziert: 3. August 2018

(c) Springer Medizin Verlag $\mathrm{GmbH}$, ein Teil von Springer Nature 2018

CrossMark

A. Nikolic · F. Rauchfuß - U. Settmacher

Klinik für Allgemein-, Viszeral- und Gefäßchirurgie, Universitätsklinikum Jena, Jena, Deutschland

\title{
Major-Leberresektionen bei zystischer Echinokokkose der Leber
}

\section{Originalpublikation}

Ramia JM, Serrablo A, Serradilla M et al (2018) Major hepatectomies in liver cystic echinococcosis: A bi-centric experience. Retrospective cohort study. Int J Surg. 54(Pt A):182-186. https://doi.org/10.1016/j.ijsu. 2018.04.049.

Hintergrund. Die durch den Parasiten Echinococcus granulosus (Hundebandwurm) hervorgerufene zystische Leberechinokokkose („liver cystic echinococcosis“, LCE) kann konservativ oder radikal chirurgisch einschließlich Leberteilresektionen bis hin zur Lebertransplantation therapiert werden. Eine Major-Leberteilresektion (mehr als drei Segmente; „major hepatectomy“, $\mathrm{MH}$ ) kann je nach Verteilung und Größe der Zysten notwendig werden. Die Erfahrungen aus zwei spanischen Zentren in einer LCE-endemischen Region sind in der Arbeit zusammengefasst.

Methodik. In der bizentrischen retrospektiven Kohortenanalyse wurden über einen Beobachtungszeitraum von 8 Jahren 145 Patienten mit gesichertem Nachweis aktiver oder komplizierter Echinococcus-granulosus-Zysten in der Leber eingeschlossen. Die Diagnose erfolgte durch Abdomencomputertomographie und Echinokokkenserologie (IgE). Bei präoperativem Ikterus und abszedierten Leberzysten wurde präoperativ eine endoskopische retrograde Cholangiopankreatikographie bzw. perkutane Drainage durchgeführt. Bei intraoperativer Zysteneröffnung folgte eine einmonatige orale Albendazoltherapie. Postoperativ erfolgte eine strukturierte
Nachsorge. Zudem wurden epidemiologische, radiologische und klinischen Daten sowie chirurgische Technik, Morbidität (Clavien-Dindo, CD I-V) und Mortalität analysiert.

Ergebnisse. Bei 49 Patienten wurde eine $\mathrm{MH}$ durchgeführt - 15 als rechte und 28 als linke Hemihepatektomie (HHE) bzw. Trisektorektomie. Bei 33 Patienten handelte es sich um die Erstdiagnose einer LCE und bei 16 um ein Rezidiv. Die Zysten waren überwiegend isoliert $(n=33)$ mit einer durchschnittlichen Größe von 9,94cm $(15-22 \mathrm{~cm})$ und meist unilobärer Verteilung $(n=43$ : rechts 27, links 16). Die Indikation für die $\mathrm{MH}$ war ein vollständiger Befall des rechten Leberlappens $(n=14)$, eine Gefäßbeteiligung (8) oder Verbindung zu den Gallenwegen $(n=9)$ sowie kombiniert $(n=18)$. Interessanterweise traten bei neudiagnostizierter LCE nach $\mathrm{MH}$ signifikant weniger, dafür schwerere Komplikationen (CD III-V, $n=13$ ) als bei intrahepatischen Rezidiven (CD I und II, $n=18$ ) auf. Minor-Komplikationen waren Bluttransfusionen $(n=12)$, Gallefisteln $(n=11)$ und entlastungswürdige Verhalte bzw. Abszesse $(n=7)$. In 12 Fällen kam es intraoperativ $\mathrm{zu}$ einer Eröffnung der Hydatiden. Vergleicht man die $\mathrm{MH}$ untereinander, traten nach rechtsseitiger HHE mit vollständigem LCE-Befall signifikant mehr Komplikationen auf. Der Krankenhausaufenthalt war signifikant länger, wenn eine Beteiligung bzw. Verbindung der LCE zu Gefäßen oder den Gallenwegen bestand. In der Nachsorge (31 Monate, 1-75 Monate) gab es keine MH-bezogenen Komplikationen und intrahepati- schen Rezidive, dafür 3 extrahepatische peritoneale Rezidive nach intraoperativer Zysteneröffnung.

\section{Fazit}

Die hier vorgestellte retrospektive Arbeit hebt an einer großen Fallzahl den Stellenwert der Leberteilresektion in der interdisziplinären Behandlung von Echinococcus-granulosus-Zysten hervor. Solche großen Fallzahlen sind nur in Endemiegebieten zu erzielen, wie es auch hier der Fall ist. Unklar bleibt allerdings, ob die antihelminthische Therapie in dieser Form adäquat war. Insbesondere nach Zysteneröffnung sollte, unseren Erfahrungen nach, eine längere Therapie erfolgen. $\mathrm{Zu}$ dem präferieren wir in unserem Zentrum eine zweimonatige „neoadjuvante“ antihelminthische Therapie vor der operativen Sanierung. Dies kann und sollte Gegenstand prospektiver Studien sein.

\section{Korrespondenzadresse}

\section{A. Nikolic}

Klinik für Allgemein-, Viszeral- und Gefäßchirurgie, Universitätsklinikum Jena Am Klinikum 1, 07747 Jena, Deutschland Alen.Nikolic@med.uni-jena.de

Interessenkonflikt. A. Nikolic, F. Rauchfuß und U. Settmacher geben an, dass kein Interessenkonflikt besteht. 